\title{
LEVEL CROSSING RATE OF SC RECEIVER OVER GAMMA SHADOWED WEIBULL MULTIPATH FADING CHANNEL
}

\author{
Suad Suljović, Dragana Krstić, Srdjan Maričić, Srboljub Zdravković, Vladeta Milenković, \\ Mihajlo Stefanović
}

Original scientific paper

The wireless telecommunication system consisting of selection combining (SC) receiver which works in Gamma shadowed Weibull multiple-faded channel is discussed in this work. The received signal suffers Weibull small scale fading which leads in variation of the signal envelope and Gamma large scale fading which results in variation of the SC receiver output signal envelope power. SC receiver is utilized to abate the impact of Gamma large scale fading effects and Weibull small scale fading effects on system characteristics. The formula for average level crossing rate (LCR) of signal envelope at SC combiner output is performed in the closed shape. The result we get can be applied to calculate the average fade duration (AFD) of such wireless systems The obtained solutions are plotted in a few graphs to point out the impact of Weibull fading envelope severity parameter and Gamma shadowing severity parameter on system features.

Keywords: Gamma shadowing; level crossing rate (LCR); selection combining (SC); Weibull fading

Srednji broj osnih presjeka kod SC prijemnika u kanalu s Weibull-ovim fedingom i Gamma sjenkom

Izvorni znanstveni članak U ovom radu je razmotren bežični telekomunikacijski sustav sa SC prijemnikom koji radi u prisustvu Weibull-ovog fedinga i Gamma sjenke. Utjecaj Weibull-ovog fedinga na primljeni signal se ogleda u promjeni anvelope signala, a utjecaj Gamma sjenke u promjeni snage izlaznog signala. SC prijemnik se koristi za smanjenje utjecaja fedinga i sjenke na karakteristike sustava. Izraz za srednji broj osnih presjeka izlaznog signala iz SC prijemnika je izveden u zatvorenom obliku. Dobiveni rezultati se mogu koristiti za izračunavanje prosječnog trajanja otkaza bežičnog sustava. Numerički rezultati su predstavljeni grafički, kako bi se prikazao utjecaj parametara fedinga i sjenke na karakteristike sustava.

Ključne riječi: broj osnih presjeka; Gamma sjenka; SC kombiniranje; Weibull-ov feding

\section{Introduction}

Multipath fading and shadowing deteriorate characteristics of wireless communication systems and restrict the channel capacity. The signal at the reception is exposed simultaneously to both large and small scale fadings. The fast fading creates the signal envelope variation and slow fading creates the variation of the signal envelope power $[1 \div 3]$.

There are several distribution which are utilized to delineate variations of signal envelope and variations of signal envelope power in fading channels. The most commonly used distributions for describing small scale variations of signal envelope are: Rician, Rayleigh, Weibull, Nakagami-m, Nakagami-q(Hoyt) and $\alpha-\mu$ distributions $[4 \div 8]$. The variation of signal envelope power is usually depicted by log-normal distribution or Gamma distribution [2].

Nakagami-m distribution and Rayleigh distribution describe short term signal envelope variations in linear, non line-of-sight multiple-faded environment. Weibull and $\alpha-\mu$ distributions are used in non linear and non line-ofsight faded medium. In linear and line-of sight channels, Rician distribution is used for a good description of short term signal envelope variation [3].

Weibull distribution has two parameters: shape parameter $\alpha, \alpha>0$, and scale parameter $\Omega, \Omega>0$ (Eq. (9)). The Weibull distribution is general distribution. Out of it, the exponential distribution can be obtained for $\alpha=1$ and the Rayleigh distribution for $\alpha=2$ [2].

In shadowed fading channels, the long term variation of signal envelope power can be inscrabed by log-normal and Gamma distributions. The modeling of variations of signal envelope power in shadowed fading channels by usage of the log-normal distribution is more suitable because it assures better matching of measurings and numerical results. On the other side, the usage of Gamma distribution allows much simpler mathematical manipulations and formulas for obtaining all system performance. More recent papers have shown that lognormal and Gamma distributions get along well $[9,10]$. The model of signal power described by Gamma distribution makes the analysis of the system easier and gives the expressions for system performance (such as probability density function (PDF) of signal to noise ratio (SNR)) in the closed shape.

Diversity techniques are widely applied methods for mitigation of the fading influence and improvement of the wireless mobile system performance without increasing the channel bandwidth or power of transmited signals. Diversity combiners combine more received signals in different ways. Maximum ratio combining (MRC) has the best performance. Selection combining (SC) is the least complicated. In SC receivers, only one of diversity branches is processed at the receiver at one moment [3].

It is important to determine the statistical characteristics of the wireless system of the first two orders. The first order statistics of a communication system are: the bit error rate (BER), the outage probability $\left(P_{\text {out }}\right)$ and the channel capacity. The second order properties of communication systems are: the joint probability density function of the signal envelope and its first derivative at the output, the average level crossing rate of signal envelope at the output and the average fade duration (AFD) [2]. 


\section{Related works}

In scientific literature many articles can be found which are engaged with the second order properties of wireless telecommunication systems working over composite shadowed multiple-faded environments $[11 \div$ 15].

First of all, in the eighties, the influence of composite Rayleigh fading was considered. The small scale Rayleigh fading acting together with large scale log-normal changes of mean value is analyzed in [11]. The interest for the investigation of influence of Rayleigh fading in wireless channels is present even today [12].

In the nineties, the log normal-shadowed Rician channels were scrutinized [13,14]. An estimation of spectrum efficiency of microcellular land mobile radio system is shown in [13] by deliberation the useful signal as fast fading with Rician distribution along the lognormal slow fading and co-channel interference as uncorrelated Rayleigh fading acting together with slow fading with log-normal distribution. Then, the microcellular system is likened to a standard macrocellular system. A new method is applied in [14] to extend earlier result by involving the effect of log-normal slow fading on the $P_{\text {out }}$ for microcellular radio systems in Rician/Rician fading environments.

Later, an analysis was done for system with macro and micro diversity receivers in shadowed fading environment. The existence of Rician fading and correlated Gamma shadowing is studied in [15]. The LCR and AFD of macro SC diversity combiner and two micro MRC diversity combiners which function in such Gamma shadowed Rician multipath fading environment are computed.

In [16], macrodiversity system with SC macro diversity combiner and two microdiversity MRC combiners working in Gamma shadowed Nakagami-m fast fading environment is investigated. The macro diversity SC combiner minimizes shadowing action on system characteristics and micro diversity MRC combiners mitigate Nakagami-m multipath fading influence to the system properties. The second-order characteristics analysis of this system is presented.

An access with infinite series for determination the system performance of a switched and stay combining (SSC) receiver with two branches, functioning in a channel with correlated Hoyt fading, was given in [17]. The evaluation is on the basis of the average bit error probability (ABEP) and the $P_{\text {out }}$.

Easy and precise formulas for characteristics of an equal gain combining (EGC) combiner with L branches, in an independent Hoyt fading environment, are obtained in [18]. By using an approximate but adequate PDF of the sum of random variables with Hoyt distribution, the formulas for the average BER for binary and both, coherent and noncoherent modulations, are performed.

The second order characteristics (average LCR and AFD) and average Shannon's channel capacity for channel with Weibull multipath fading are acquired in [19] as formulas in the closed form. In [20], the formulas for the average LCR and AFD at the output of SC combiner with two branches are obtained. In that paper, the authors reviewed and compared the results from some previously published papers in a more general way.

In later papers the shadowing is taken into account. In such a way, the paper [21] treats second order characteristics of the signal envelope in the receiver in mobile radio channels under the influence of Weibull fading and shadowing. The output signal is represented as the product of Weibull and log-normal process. The mathematical solutions for LCR and AFD for signal envelope are derived. The parameters of fading and shadowing affecting this statistics are shown in several graphs.

In frequency non-selective fading environments, the waves that occur in receiver are not homogeneous because of nonuniform scattering from the objects of reflective nature. Besides, large scale fading affects the level of received signal by inducing slow variations to its mean value. In [22], the authors studied propagation taking into account both, inhomogeneous scattering and shadowing, by multiplication of the Weibull process and log-normal process. The Weibull one includes scattering nonuniformities of the environment, while log-normal process takes into account the slow term changes of the mean value because of shadowing. The correct expression for the composite PDF is provided as well as approximate expressions for the second order characteristics (LCR and ADF).

The level crossing rates of SC receiver output signal are set by these authors in [23] for two examples: 1) the presence of Gamma shadowing and Rician multipath fading; 2) the presence of Gamma shadowing and $k-\mu$ multipath fading. In this article, the wireless telecommunication system with one SC combiner performing in Gamma shadowing and multipath fading channel will be discussed also, but the signal in channel being affected at the same time by Gamma slow fading and Weibull fading. Weibull multipath fading creates variations of signal envelope and Gamma slow fading creates variations of signal envelope power.

The joint PDF of SC combiner output signal envelope and its first derivative is obtained by using the joint PDF of signals and their first derivatives on the inputs of SC combiner. The average LCR of SC combiner output signal envelope is deffined as the average value of the first derivative of SC combiner output signal envelope [2]. The SC combiner is used to alleviate the multipath fading and shadowing impacts on system performance. After obtaining the expression for average LCR of SC combiner output signal in the closed form, the results are depicted in some figures.

By the author's knowledge, the second order characteristics of wireless telecommunication system with SC combiner which acts in Gamma shadowed Weibull fading medium is not published in the available scientific literature.

\section{Level crossing rate}

The samples of Weibull random process can be obtained by nonlinear transformation of samples of Rayleigh random process. Weibull random variable is [2]:

$x=y^{\frac{z}{\alpha}}$, 
where $y$ is Rayleigh random variable. Squared Rayleigh random variable is a summation of two squared random variables with Gaussian distribution [1]:

$x^{\propto}=y^{z}=y_{1}{ }^{2}+y_{2}{ }^{2}$,

with $y_{1}$ and $y_{2}$ being independent, zero mean, random variables with Gaussian distribution and same variances $\sigma^{2}$.

The first derivative of Weibull random variable is:

$\dot{x}=\frac{1}{\propto x^{\alpha-1}}\left(y_{1} \dot{y}_{1}+y_{2} \dot{y}_{2}\right)$.

The first derivation of random variable with Gaussian distribution is random variable with Gaussian distribution. These variables $\dot{y}_{1}$ and $\dot{y}_{2}$ are random variables with Gaussian distribution. The random variables with Gaussian distribution have linear transformation with Gaussian distribution. Hence, the first derivative of Weibull random variable has conditional Gaussian distribution. The average value of $\dot{x}$ is:

$\overline{\dot{x}}=\frac{1}{\alpha x^{\alpha-1}}\left(y_{1} \overline{\dot{y}_{1}}+y_{2} \overline{\dot{y}_{2}}\right)=0$,

since $\overline{\dot{y}_{1}}=\overline{\dot{y}_{2}}=0$.

The variance of $\dot{x}$ is:

${\sigma_{\dot{x}}}^{2}=\frac{1}{\alpha^{2} x^{2 \alpha-2}}\left(y_{1}{ }^{2}{\sigma_{\dot{y}_{1}}}^{2}+y_{2}{ }^{2}{\sigma_{\dot{y}_{2}}}^{2}\right)$,

where

${\sigma_{\dot{y}_{1}}}^{2}={\sigma_{\dot{y}_{2}}}^{2}=2 \pi^{2} \sigma^{2} f_{m}{ }^{2}=\pi^{2} f_{m}{ }^{2} \Omega$,

and $f_{m}{ }^{2}$ is maximal Doppler frequency.

If we replace the expression (6) in the expression (5), the variance is obtained as:

$$
\begin{aligned}
& {\sigma_{\dot{x}}}^{2}=\frac{1}{\alpha^{2} x^{2 \alpha-2}} \pi^{2} f_{m}{ }^{2} \Omega\left(y_{1}{ }^{2}+y_{2}{ }^{2}\right)= \\
& =\frac{1}{\alpha^{2} x^{2 \alpha-2}} \pi^{2} f_{m}{ }^{2} \Omega \mathrm{x}^{\alpha}=\frac{\pi^{2} f_{m}{ }^{2} \Omega}{\alpha^{2} x^{2 \alpha-2}} .
\end{aligned}
$$

The joint PDF of Weibull random envelope and its first derivative is:

$P_{x \dot{x}}(x \dot{x})=P_{\dot{x}}(\dot{x} / x) P_{x}(x)$,

where $P_{x}(x)$ is PDF of Weibull random envelope [2]:

$P_{x}(x)=\frac{\alpha}{\Omega} x^{\alpha-1} e^{-\left(\frac{1}{\Omega} x^{\alpha}\right)}$.

After substituting (9) in (8), the expression for joint PDF of Weibull random envelope and the first derivative of Weibull random envelope becomes:

$P_{x \dot{x}}(x \dot{x})=\frac{1}{\sqrt{2 \pi} \sigma_{\dot{x}}} e^{-\left(\frac{\dot{x}^{2}}{2 \sigma \dot{x}^{2}}\right)} \frac{\alpha}{\Omega} x^{\alpha-1} e^{-\left(\frac{1}{\Omega} x^{\alpha}\right)}$.
The LCR of Weibull random envelope shall be computed as an average value of the first derivative of Weibull random envelope [2]:

$N_{x}=\int_{0}^{\infty} d \dot{x} \dot{x} P_{x \dot{x}}(x \dot{x})=$

$=\frac{\alpha}{\Omega} x^{\alpha-1} e^{-\left(\frac{1}{\Omega} x^{\alpha}\right)} \int_{0}^{\infty} d \dot{x} \dot{x} \frac{1}{\sqrt{2 \pi} \sigma_{\dot{x}}} e^{-\left(\frac{\dot{x}^{2}}{2 \sigma \dot{x}^{2}}\right)}=$

$=\frac{\pi f_{m}}{\sqrt{2 \pi}} \frac{x^{\alpha / 2}}{\Omega^{1 / 2}} e^{-\left(\frac{1}{\Omega} x^{\alpha}\right)}$

In Gamma shadowed Weibull multipath fading environment, the signal envelope power $\Omega$ has Gamma distribution:

$P_{\Omega}(\Omega)=\frac{1}{\beta^{c} \Gamma(c)} \Omega^{c-1} e^{-\left(\frac{1}{\beta} \Omega\right)}$.

In this case, the average LCR can be computed from the expression [24]:

$N_{x}=\int_{0}^{\infty} \mathrm{d} \Omega n x / \Omega P_{\Omega}(\Omega)=$

$=\int_{0}^{\infty} \mathrm{d} \Omega \frac{\pi f_{m}}{\sqrt{2 \pi}} \frac{x^{\alpha / 2}}{\Omega^{1 / 2}} e^{-\left(\frac{1}{\Omega} x^{\alpha}\right)} \frac{1}{\beta^{c} \Gamma(c)} \Omega^{c-1} e^{-\left(\frac{1}{\beta} \Omega\right)}=$

$=\frac{\pi f_{m}}{\sqrt{2 \pi}} x^{\alpha / 2} \frac{1}{\beta^{c} \Gamma(c)} \int_{0}^{\infty} \mathrm{d} \Omega \Omega^{c-1-1 / 2} e^{-\left(\frac{x^{\alpha}}{\Omega}-\frac{\Omega}{\beta}\right)}=$

$=\frac{\pi f_{m}}{\sqrt{2 \pi}} \frac{1}{\beta^{c} \Gamma(c)} x^{\alpha / 2}\left(x^{\alpha} \beta\right)^{\frac{c}{2}-\frac{1}{4}} K_{c-1 / 2}\left(2 \sqrt{\frac{x^{\alpha}}{\beta}}\right)$

where $K_{n}(x)$ marks the modified Bessel function of the second kind [25, eq. (3.471.9)].

By means of this formula, AFD of wireless telecommunication system working over Gamma shadowed Weibull fast fading medium can be calculated.

Now, the wireless telecommunication system with dual SC combiner under the action of Gamma shadowing and Weibull multipath fading is considered. The joint PDF of SC combiner output signal envelope and its first derivative is:

$P_{z \dot{z}}(z \dot{z})=P_{z_{1} \dot{z}_{1}}(z \dot{z}) F_{z_{2}}(z)+P_{z_{2} \dot{z}_{2}}(z \dot{z}) F_{z_{1}}(z)=$

$=2 P_{z_{1} \dot{z}_{1}}(z \dot{z}) F_{z_{2}}(z)$,

with $z$ being SC combiner output signal envelope and $z_{1}$ and $z_{2}$ are envelopes of input signals in SC combiner, and

$F_{z_{2}}(z)=1-e^{-\left(\frac{1}{\beta} z^{\alpha}\right)}, \quad z \geq 0$,

is cumulative distribution function (CDF) of $z$. 
After substitutions (10) and (15) in (14), the formula for the joint PDF of selection combiner output signal envelope and the first derivation becomes:

$P_{z \dot{z}}(z \dot{z})=z \frac{1}{\sqrt{2 \pi} \sigma_{\dot{\chi}}} e^{-\left(\frac{\dot{z}^{2}}{2 \sigma_{\dot{x}}^{2}}\right)} \frac{\alpha}{\Omega} z^{\alpha-1} e^{-\frac{1}{\Omega} z^{\alpha}}\left(1-e^{-\frac{1}{\beta} z^{\alpha}}\right)$

The LCR of SC combiner output signal is:

$N_{z}=\int_{0}^{\infty} \mathrm{d} \dot{z} \dot{z} P_{z \dot{z}}(z \dot{z})=$

$=\frac{2 \alpha}{\Omega} z^{\alpha-1} e^{-\left(\frac{1}{\Omega} z^{\alpha}\right)}\left(1-e^{-\left(\frac{1}{\beta} z^{\alpha}\right)}\right)$.

$\cdot \int_{0}^{\infty} \mathrm{d} \dot{z} \dot{z} \frac{1}{\sqrt{2 \pi} \sigma_{\dot{x}}} e^{-\left(\frac{\dot{z}^{2}}{2 \sigma_{\dot{x}}^{2}}\right)}=$

$=\frac{2 \alpha}{\Omega} z^{\alpha-1} e^{-\left(\frac{1}{\Omega} z^{\alpha}\right)}\left(1-e^{-\left(\frac{1}{\beta} z^{\alpha}\right)}\right) \frac{\pi f_{m} \Omega^{1 / 2}}{\sqrt{2 \pi} z^{\alpha} / 2^{-1} \alpha}=$

$=\frac{2 \pi f_{m}}{\sqrt{2 \pi}} \frac{z^{\alpha / 2}}{\Omega^{1 / 2}} e^{-\left(\frac{1}{\Omega} z^{\alpha}\right)}\left(1-e^{-\left(\frac{1}{\beta} z^{\alpha}\right)}\right)$.

The power of Weibull signal envelope is Gamma distributed. The LCR of SC combiner output signal envelope in Gamma shadowed Weibull short term fading environment is:

$N_{z}=\int_{0}^{\infty} \mathrm{d} \Omega N_{z / \Omega} P_{\Omega}(\Omega)=$

$=\int_{0}^{\infty} \frac{2 \pi f_{m}}{\sqrt{2 \pi}} \frac{z^{\alpha / 2}}{\Omega^{1 / 2}} e^{-\left(\frac{1}{\Omega} z^{\alpha}\right)}$.

$\cdot\left(1-e^{-\left(\frac{1}{\Omega} z^{\alpha}\right)}\right) \frac{1}{\beta^{c} \Gamma(0)} \Omega^{c-1} e^{-\left(\frac{1}{\beta} \Omega\right)}=$

$=\frac{f_{m} \sqrt{2 \pi}}{\beta^{c} \Gamma(0)} z^{\frac{\alpha}{2}} \int_{0}^{\infty} \mathrm{d} \Omega \Omega^{c-1-\frac{1}{2}}\left(e^{-\left(\frac{1}{\Omega} z^{\alpha}\right)}-e^{-\left(\frac{2}{\Omega} z^{\alpha}\right)}\right) e^{-\left(\frac{1}{\beta} \Omega\right)}=$

$=\frac{f_{m} \sqrt{2 \pi}}{\beta^{c} \Gamma(0)} z^{\frac{\alpha}{2}} \int_{0}^{\infty} \mathrm{d} \Omega \Omega^{c-1-\frac{1}{2}} \cdot\left(e^{-\left(\frac{z^{\alpha}}{\Omega}-\frac{\Omega}{\beta}\right)}-e^{-\left(\frac{2 z^{\alpha}}{\Omega}-\frac{\Omega}{\beta}\right)}\right)=$

$=\frac{f_{m} \sqrt{2 \pi}}{\beta^{c} \Gamma(0)} z^{\frac{\alpha}{2}}\left(\left(\beta z^{\alpha}\right)^{\frac{c}{2}-\frac{1}{4}} K_{c-1 / 2}(2 \sqrt{2 \alpha / \beta})\right)\left(2 \beta z^{\alpha}\right)^{\frac{c}{2}-\frac{1}{4}}$.

$\cdot K_{c-1 / 2}\left(2 \sqrt{2 z^{\alpha} / \beta}\right)=\frac{f_{m} \sqrt{2 \pi}}{\beta^{c} \Gamma(0)} z^{\alpha / 2}\left(\beta z^{\alpha}\right)^{\frac{c}{2}-\frac{1}{4}}$.

$\cdot\left(K_{c-1 / 2}\left(2 \sqrt{\frac{z^{\alpha}}{\beta}}\right)-z^{\frac{c}{2}-\frac{1}{4}} K_{\mathrm{c}-1 / 2}\left(2 \sqrt{\frac{2 z^{\alpha}}{\beta}}\right)\right)$.

\section{$4 \quad$ Numerical results}

The average LCR of SC combiner output signal envelope is given depending on the SC combiner output signal envelope for a number of values of Weibull multipath fading severity parameter $\alpha$ ( $a$ in figures), Gamma slow fading severity parameter $c$ and average power of Gamma random variable $b$ in Fig. 1 .

The average LCR increasing for smaller values of SC combiner output signal envelope $z$, reaches the maximum, then decreases. The impact of SC combiner output signal envelope on average LCR is greater for higher values of Weibull multipath fading severity parameters. Average
LCR has lower values for bigger values of Weibull multipath fading severity parameter and then system performance is better.

In Fig. 2, the average LCR is plotted versus Gamma shadowing severity parameter $c$ for a few magnitudes of SC combiner output signal envelope, Weibull multipath fading severity parameter $\alpha$, and average power of Gamma random variable $b$.

Average LCR increases as Gamma shadowing severity parameter grows. For bigger dimensions of Weibull multipath severity parameter $\alpha$, average level crossing rate has lower sizes. Also, average LCR has lower values for greater values of average power of Gamma random variable $b$ and $\mathrm{SC}$ combiner output signal envelope $z$.

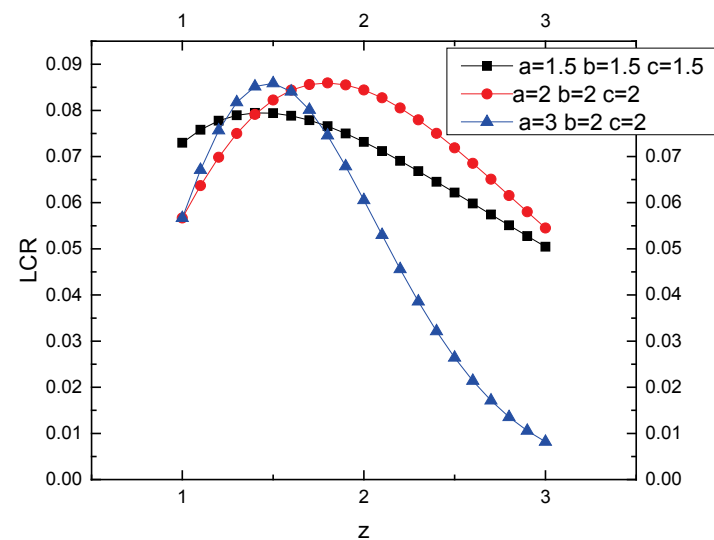

Figure 1 LCR versus signal envelope

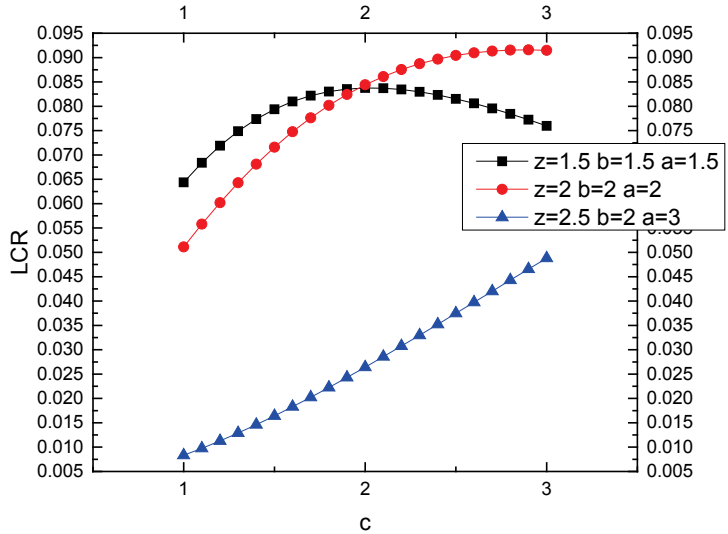

Figure 2 LCR versus shadowing severity

The system properties are more favorable for smaller values of average LCR. The obtained results are drawn to explain the impact of Weibull multipath fading severity parameter and Gamma slow fading severity parameter on average LCR.

The best performance is achieved with a small average LCR, namely with bigger SC combiner output signal envelope $z$, Weibull multipath fading severity parameter $\alpha$, average power of Gamma random variable $b$ and with declining of Gamma shadowing severity parameter $c$.

\section{Conclusion}

The wireless telecommunication system with SC combiner with two branches, working over channel under 
presence of slow and fast fading is analyzed. The received signal suffers Gamma shadowing and Weibull fading leading in system performance impairment. Weibull small scale fading triggers off variation of the signal envelope and Gamma large scale fading induces changes of the signal envelope power. SC combiner is used for reducing the long and short term fading influences on system properties.

In this paper, the useful formula for the average LCR of SC combiner output signal envelope in the closed form is determined. This expression was found as an average value of the first derivative of the SC combiner output signal envelope. The joint PDF of SC combiner output signal envelope and its first derivative is also established.

The average LCR grows for small values of SC combiner output signal envelope and declines for bigger values of SC combiner output signal envelope. For lower values of Weibull multipath fading severity parameter and lower values of Gamma shadowing severity parameter SC receiver output has a larger impact on the average LCR.

Therefore, for better system performance it is good to choose higher values for: SC combiner output signal envelope $z$,Weibull multipath fading severity parameter $\alpha$, and average power of Gamma random variable $b$ and lower for Gamma shadowing severity parameter $c$.

The obtained results areuseful in performance analyzing and production of wireless telecommunication systems with SC combiners which work per channels under the effects of Gamma shadowing and Weibull fading. By selecting optimal parameters, the best performance could be achieved and the reliable systems obtained

\section{Acknowledgements}

This article is realised in the framework of projects TR-33035 and III-44006 of the Ministry of Education, Science and Technological Development of Serbia.

\section{References}

[1] Proakis, J. Digital Communications, $4^{\text {th }}$ ed. New York: McGraw-Hill, 2001

[2] Simon, M. K.; Alouini, M. S. Digital Communication over Fading Channels, Wiley: New York, 2000. DOl: 10.1002/0471200697

[3] Shankar, M. Fading and Shadowing in Wireless Systems, Technology \& Engineering, ISBN 978-1-4614-0366-1, eISBN 978-1-4614-0367-8, Springer, New York, Dordrecht, Heidelberg, London, 2011. DOI: 10.1007/978-1-4614-0367-8

[4] Rice, O. S. Mathematical Analysis of Random Noise, Dover, New York, 1945.

[5] Weibull, W. A statistical distribution function of wide applicability. // J. Appl. Mech.-Trans. ASME. 18, 3(1951), pp. 293-297.

[6] Nakagami, M. The m-distribution, a general formula of intensity distri-bution of rapid fading,in Statistical Methods in Radio Wave Propagation, Pergamon Press, (1960), pp. 336. DOI: 10.1016/B978-0-08-009306-2.50005-4

[7] Paris J. F. Nakagami-q (Hoyt) distribution function with applications. // Electronics Letters. 45, 4(2009), pp. 210211. DOI: 10.1049/el:20093427

[8] Yacoub, M. D. The $\alpha-\mu$ Distribution: A Physical Fading Model for the Stacy Distribution. // IEEE Transactions on
Vehicular Technology. 56, 1(2007), pp. 27-34. DOI: 10.1109/TVT.2006.883753

[9] Kostic, I. Analytical approach to performance analysis for channel subject to shadowing and fading. // IEEE Proc. Comm. 152, (2005), pp. 821-827.

[10] Sekulovic, N. M.; Stefanovic, M. C. Performance analysis of system with micro- and macrodiversity reception in corelated gamma shadowed Rician fading channels. // Wireless Personal Commun. 65, 1(2012), pp. 143-156. DOI: 10.1007/s11277-011-0232-8

[11] Hansen, F.; Meno, F. I. Mobile Fading-Rayleigh and Lognormal Superimposed. // IEEE Transactions on Vehicular Technology. VT-26, 4(1977), pp. 332-335. DOI: 10.1109/T-VT.1977.23703

[12] Gomez-Deniz, E.; Gomez-Deniz, L. A generalisation of the Rayleigh distribution with applications in wireless fading channels. // Wireless Communications and Mobile Computing, Published online 8 February 2011 in Wiley Online Library. 13, 1(2013), pp. 85-94. DOI: 10.1002/wcm. 1097

[13] Prasad, R.; Kegel, A. Effects of Rician faded and lognormal shadowed signals on spectrum efficiency in microcellular radio. // IEEE Transactions on Vehicular Technology. 42, 3(1993), pp. 274-281. DOl: 10.1109/25.231878

[14] Tjhung,T. T.; Choy, C. C.; Dong, X. Outage probability for lognormal-shadowed Rician channels. // IEEE Transactions on Vehicular Technology. 46, 2(1997), pp. 400-407. DOI: 10.1109/25.580779

[15] Sekulović, N. M.; Stefanović, M. Č. Performance Analysis of System with Micro- and Macrodiversity Reception in Correlated Gamma Shadowed Rician Fading Channels. // Wireless Personal Communications. 65, 1(2012), pp. 143156. DOI: $10.1007 / \mathrm{s} 11277-011-0232-8$

[16] Stefanovic, D.; Panic, S. R.; Spalevic, P. Second-order statistics of SC macrodiversity system operating over Gamma shadowed Nakagami-m fading channels. // AEU International Journal of Electronics and Communications. 65, 5(2011), pp. 413-418. DOI: 10.1016/j.aeue.2010.05.001

[17] Bandjur, M. V.; Bandjur, Dj. V. Performance Analysis of SSC Diversity Receiver over Correlated Hoyt Fading Channels. // Radioengineering. 21, 1(2012), pp. 110-114.

[18] Baid, A.; Fadnavis, H.; Sahu, P. R. Performance of a Predetection EGC Receiver in Hoyt Fading Channels for Arbitrary Number of Branches. // IEEE Communication Letters. $\quad 12, \quad 9(2008), \quad$ pp. 627-629. DOI: 10.1109/LCOMM.2008.080297

[19] Sagias, N. C.; Zogas, D. A.; Karagiannidis, G. K.; Tombras G. S. Channel Capacity and Second-Order Statistics in Weibull Fading. // IEEE Communication Letters. 8, 6(2004), pp. 377-379. DOI: 10.1109/LCOMM.2004.831319

[20] Sagias, N. C.; Karagiannidis, G. K. Comments on "Average LCR and AFD for SC diversity over correlated Weibull fading channels". //Wireless Personal Communications. 43, 2(2007), pp. 699-701. DOI: 10.1007/s11277-007-9274-3

[21] Mitic, A. M.; Jakovljevic, M. M. Second-Order Statistics in Weibull-Lognormal Fading Channels. $/ / 8^{\text {th }}$ International Conference on Telecommunications in Modern Satellite, Cable and Broadcasting Services, TELSIKS 2007. Nis, 2628 Sept. 2007. DOI: 10.1109/TELSKS.2007.4376062

[22] Karadimas, P.; Kotsopoulos, S. A. The Weibull-Lognormal Fading Channel: Analysis, Simulation, and Validation. // IEEE Transactions on Vehicular Technology. 58, 7(2009), pp. 3808-3813. DOI: 10.1109/TVT.2008.2002697

[23] Krstic, D. S.; Suljovic, S.; Stefanovic, M. C.; Yassein, M. M. B.; Aleksic, D. Level Crossing Rate of SC Receiver Output Signal in the Presence of Gamma Shadowing and k$\mu$ or Rician Multipath Fading. // International Journal of Communications. 9, (2015), pp. 19-27. 
[24] Gradshteyn, I.; Ryzhik, I. Tables of Integrals, Series, and products. Academic Press, New York 1994.

[25] Modified Bessel Function of the Second Kind, http://mathworld.wolfram.com/ModifiedBesselFunctionoft heSecondKind.html

\section{Authors' addresses}

Suad Suljović

Faculty of Electronic Engineering, University of Niš,

Aleksandra Medvedeva 14, 18000 Niš, Serbia

E-mail: suadsara@gmail.com

Dragana Krstić

Faculty of Electronic Engineering, University of Niš, Aleksandra Medvedeva 14, 18000 Niš, Serbia

Fax: +38118588399

E-mail: dragana.krstic@elfak.ni.ac.rs

Srdjan Maričić

Faculty of Industrial Management, University Union

Belgrade, Kosančićev venac 2, 11000 Belgrade, Serbia

E-mail: srdjan.maricic@gmail.com

\section{Srboljub Zdravković}

Faculty of Electronic Engineering, University of Niš,

Aleksandra Medvedeva 14, 18000 Niš, Serbia

E-mail: srba82@gmail.com

\section{Vladeta Milenković}

Faculty of Electronic Engineering, University of Niš,

Aleksandra Medvedeva 14, 18000 Niš, Serbia

E-mail: vladeta.milenkovic@elfak.ni.ac.rs

\section{Mihajlo Stefanović}

Faculty of Electronic Engineering, University of Niš,

Aleksandra Medvedeva 14, 18000 Niš, Serbia

E-mail: misa.profesor@gmail.com 